\title{
Reducing the use of plain abdominal radiographs in an emergency department
}

\author{
G. A. JELINEK \& N. D. G. BANHAM
}

Department of Emergency Medicine, Fremantle Hospital, Fremantle, Western Australia

\section{SUMMARY}

Plain abdominal radiographs are ordered frequently in emergency departments. Previous studies have shown that these radiographs are often requested inappropriately with little likelihood of producing useful information. This study shows that the overall number can be reduced significantly, with little risk to patients, when clear guide-lines are applied.

\section{INTRODUCTION}

The plain abdominal radiograph (PAR) is frequently used to assist diagnosis in the emergency department. Several studies have suggested that it is used excessively, and often inappropriately.

In a retrospective series of 100 consecutive cases, de Lacey et al., (1980) showed that $78 \%$ of PARs were negative. A prospective study of 100 cases by McCook et al. (1982) showed very similar results, with $77 \%$ of films normal, increasing to $84 \%$ if unsuspected and unrelated findings were taken into account.

Other reports concur that PARs requested without specific indications are likely to be negative. Hayward et al. (1984) and Greene (1986) concluded that there is a need to determine specific indications for PARs. Greene further suggested that the numbers may be reduced by at least $50 \%$ if his recommendations for the appropriate use of PARs in the emergency department were followed.

This study was planned with the aim of reducing the number of PARs requested in the Fremantle Hospital emergency department by applying clear guide-lines, and to determine whether such a reduction would affect the number of positive findings.

Correspondence: Dr. G. A. Jelinek. Department of Entergency Medicine, Fremantle Hospital, PO Box 480, Fremantle, Western Australia 6160 


\section{METHOD}

A PAR at Fremantle Hospital is a single supine abdominal film from the diaphragms to the hernial orifices. An erect film may be requested in addition; if it is not possible to perform an erect film, a lateral decubitus film is taken.

For the first 6 months of this study, no attempt was made to alter the pattern of requesting PARs and the medical staff were unaware of the study.

For the second 6 months, guide-lines were issued for requesting PARs, and medical staff were required to check with a consultant or registrar before such a request. Authorization was granted if the suspected condition fell into one of the categories in Table 1 . These categories were chosen by the authors after a review of the literature and discussion concerning their own clinical practice.

The radiology secretary recorded the total number of PARs in the 12 months. The clerical staff in Radiology were asked to return a photocopy of all request forms for PARs performed to the Emergency Department. One author (G.A.J.) checked the radiologist's report of all those returned and coded the forms.

Radiographs were classified 'appropriate' if the history and examination recorded on the request form led to a clinical diagnosis of one of the conditions in Table 1, and 'inappropriate' if they did not.

Abdominal ultrasonography was advised for investigation of suspected biliary tract disease or abdominal aortic aneurysm.

\section{RESULTS}

In all, 328 of $699(47 \%)$ request forms and reports were returned in the first 6 months, and 175 of $334(52 \%)$ in the second. The results are recorded in Table 2. In the first 6 months, 699 PARs were performed. This fell to 334 in the second 6 months, a drop of over $50 \%$.

Approximately half of all PARs in the first 6 months were considered inappropriate, falling to about one third in the second half of the study.

The percentage of positive findings rose from $12 \%$ to $17 \%$ from the first to second 6 months, but of those films appropriately ordered the percentage of positive findings changed little ( $25 \%$ to $26 \%$ ).

Table 3 illustrates some of the conditions for which PAR's were requested inappropriately. Although some of these conditions are occasionally associated with suggestive radiographic findings, these findings rarely alter management.

Table 1. Appropriate conditions for requesting a PAR.

Bowel obstruction or paralytic ileus

Pneumoperitoneum

Renal/ureteric/bladder calculus

Ingested foreign body

Penetrating injury 
Table 2. Analysis of PAR's in the Emergency Department over 12 months.

\begin{tabular}{lcc}
\hline & First 6 months & Second 6 months \\
\hline Total & 699 & 334 \\
Appropriate & $51 \%$ & $67 \%$ \\
Inappropriate & $49 \%$ & $33 \%$ \\
Positive findings & $12 \%$ & $17 \%$ \\
Positive findings (of those & & \\
appropriately ordered) & $25 \%$ & $26 \%$ \\
\hline
\end{tabular}

Table 3. Conditions for which a PAR was

requested inappropriately.

Haematemesis

Peptic ulceration

Renal carbuncle

Appendicitis

Pancreatitis

Giardiasis

Gastroenteritis

Ascites

Constipation

Chronic left iliac fossa pain

Anxiety

Vaginal discharge

Back pain

Diarrhoea

Hiatus hernia

Renal contusion

Lymphoma

Gastric erosions

Fractured rib

The presence of a faecolith in suspected appendicitis is an example. Such conditions were considered inappropriate indications.

\section{DISCUSSION}

The Emergency Department at Fremantle Hospital has some 82000 occasions of service annually. In the first 6 months of this study, there were 699 PARs requested. In the second 6 months, after the implementation of guide-lines, there were less than 350 , a reduction of over $50 \%$. Although the overall number of radiographs dropped by half, a third of those ordered in the second half of the study were still inappropriate. It seems there were also fewer appropriate PARs in this period. Why this should occur is difficult to understand, but it appears that the drop in 
overall numbers was not simply by chance, as the monthly numbers during the first 6 months were fairly constant, as they were during the second 6 months but at about half the previous rate. Perhaps doctors, being aware of the study were less likely to request radiography even in cases previously considered appropriate, such as renal colic, where they were able to avoid such a request by using alternative investigations, such as intravenous urography or ultrasound.

Several papers have indicated that positive findings are very unlikely to be detected on inappropriately ordered PARs. This study confirms that view. In the first 6 months, there were seven positive findings in those radiographs which were requested inappropriately. Three were unsuspected, asymptomatic gallstones. There was one unsuspected renal stone, one abdominal soft tissue mass, and 2 unsuspected bowel obstructions. On scrutiny of the notes in the latter four cases, failure to make the diagnosis clinically prior to abdominal radiography resulted from deficiencies in history or examination.

In the second 6 months none of the inappropriately requested radiographs had positive findings.

Although the total number of positive findings fell in the second 6 months, it is important to note that the proportion of positive findings found in those PARs requested appropriately remained unchanged at about a quarter. This would indicate that the drop in total numbers of PAR's did not result in a failure to detect positive findings. It could be argued that a radiograph which does not detect positive findings is of value in excluding certain conditions, and therefore a judgement of whether a PAR is of value simply by whether it detects positive findings is not valid. We concur with this view but feel that the exclusion of those conditions in Table 1, if they are suspected clinically, is quite appropriate, but in other conditions, such as those in Table 3, a PAR is of little value.

There are several advantages in reducing the number of inappropriately ordered PARs in the Emergency Department. Selective use of abdominal radiography is time efficient, uses limited staff to the best advantage and maintains the enthusiasm of the staff concerned. In a busy emergency department, delays produced by unnecessary radiography limit the attention available for other patients. At night particularly, radiology department staff rightly resent performing films with little likelihood of producing useful information. More easily quantified is the financial benefit one could expect. The Medicare rebate for a PAR at the time of the study was $A \$ 39.50$. Reducing the number of these radiographs by over 700 annually produces a saving of over $\$ 27650$. Although this calculation is of course not entirely valid in a hospital setting, it does indicate that substantial savings may be made. Stewart et al. (1985) have estimated a cancer risk of one death, or two cases per 140000 films, due to radiation from a PAR. Moreover, many patients have PARs performed on several occasions. Lowering the number of PARs reduces this risk.

It is possible to further reduce the number of PARs. When a pneumoperitoneum is suspected, Miller et al. (1971) argue that a well penetrated erect chest film is more appropriate. In suspected ureteric colic Roth et al. (1985) conclude that the PAR provides no diagnostic information that cannot be obtained by history, examination and urinalysis. If further investigation is required then excretary 
urography is the correct investigation. Management decisions are rarely made on the basis of PAR alone in acute ureteric colic and therefore it is illogical to submit the patient to a PAR when this is undertaken as a preliminary film before a contrast study. Although there was a large reduction in the number of inappropriate PARs there remain a number which may be reduced further with better selection on clinical grounds and closer supervision.

\section{ACKNOWLEDGEMENTS}

We would like to thank Nicole Jenner and Shirley Stubber for their assistance in copying and returning the request forms, and Elaine Sillery and Lee Jelinek for preparing the manuscript.

\section{REFERENCES}

de Lacey G. J., Wignall B. K., Bradbrooke S., et al. (1980) Rationalizing abdominal radiography in the accident and emergency department. Clinical Radiology 31, 453-5.

Greene C. S. (1986) Indications for plain abdominal radiography in the emergency department. Annals of Emergency Medicine 15, 257-60.

Hayward W. M. J., Hayward C., Ennis W. R., et al. (1984) A pilot evaluation of radiography in the acute abdomen. Clinical Radiology 35, 289-91.

McCook T. A., Ravin C. E. \& Rice R. P. (1982) Abdominal radiography in the emergency department. A prospective analysis. Annals of Emergency Medicine 11, 7-8.

Miller R. E. \& Nelson S. W. (1971) The roentgenologic demonstration of tiny amounts of free peritoneal gas: Experimental and clinical studies. American Journal of Roentgenology, Radium Therapy and Nuclear Medicine 112, 574-585.

Roth C. S., Bowyer B. A. \& Berquist T. H. (1985) Utility of the plain radiograph for diagnosing ureteral calculi. Annals of Emergency Medicine 14, 311-5.

Stewart A., Webb J. \& Hewitt D. (1985) A survey of childhood malignancies. British Medical Journal 1, 1495-1508. 